\title{
Geoelectrical modeling of shallow structures using parallel and perpendicular arrays
}

\author{
Néstor Bonomo*, Ana Osella*, and Patricia Martinelli* \\ Dto. de Física, Fac. Cs. Exactas y Naturales, Universidad de Buenos Aires, Ciudad Universitaria, Pab.1, 1428 Buenos Aires, Argentina
}

(Received December 5, 2000; Revised October 2, 2001; Accepted November 6, 2001)

\begin{abstract}
In this article we analyze the sensitivity of a geoelectrical modeling technique to image 2D shallow structures. Firstly, we extend a previously developed 2D method based on Rayleigh-Fourier expansions, in order to allow arbitrary locations for the electrodes and also 3D earth models. This method is an alternative to finite element and finite difference techniques and is especially suitable to model multilayered structures, with smooth irregular boundaries. Then, for simple 2D models we build up two synthetic pseudosections, one for electrode deployments parallel to a profile perpendicular to the strike, and other for deployments perpendicular to it. We analyze the advantages in using both pseudosections to model these structures. We also compare geoelectric results with the corresponding audiomagnetotelluric transverse electric and transverse magnetic responses. Finally, we perform a geoelectrical survey to image a shallow buried structure and show the goodness of the model fit obtained considering both pseudosections. For the examples studied here, we conclude that considering both pseudosections leads to a more accurate description of the structures. When a 2D anomaly is present, its effect on the perpendicular component is more focused, both in width and depth, than in the parallel component. Hence the perpendicular component helps to constrain the localization of the inhomogeneity. In addition, we find similarities between the geoelectric parallel and perpendicular responses and the corresponding audiomagnetotelluric transverse magnetic and transverse electric results, respectively. When inverting audiomagnetotelluric data using 2D codes, better resolution in the electrical imaging is obtained when both modes are considered; then it is expected that 2D imaging of geoelectric data including both arrays should lead to an optimization of the inversion process. Even more, if results of these inversions could be used in correlation with AMT results, it is clear that this kind of joint inversion should contribute to remove uncertainties allowing an improvement in the description of the actual structures.
\end{abstract}

\section{Introduction}

Archaeological excavation strategies have changed dramatically in the last decade. The application of non-invasive methods are becoming increasingly important to characterize archaeological sites. In many cases, cultural resource management and site conservation prevent excavation in large areas, and remote sensing methods must therefore be employed to obtain subsurface information.

Among the different geophysical methods, electrical resistivity surveys are specially useful for mapping the areal extent of buried foundations. Many studies have been done in this field in different parts of the world (Noel and Xu, 1991), but up to now these techniques have not been applied in Argentina for archaeological exploration. Then, as a first step to the characterization of an archaeological site in South Argentina, we focused our study to analyze the sensitivity of two-dimensional (2D) geoelectrical modeling techniques to image shallow structures, usually resistive with respect to the surrounding medium.

Both forward and inversion DC methods have been im-

*Also at CONICET (Consejo Nacional de Investigaciones Científicas y Técnicas).

Copy right (c) The Society of Geomagnetism and Earth, Planetary and Space Sciences (SGEPSS); The Seismological Society of Japan; The Volcanological Society of Japan; The Geodetic Society of Japan; The Japanese Society for Planetary Sciences. plemented, and applied to different array configurations (Queralt et al., 1991; Park and Van, 1991; Oldenburg et al., 1993; Oldenburg and Li, 1994; Zhang et al., 1995; Spitzer, 1995; Loke and Barker, 1996a, b). At present, the usual practice to map shallow structures is to perform a survey along a profile assumed to be perpendicular to a suspected strike axis, with electrodes deployed along this direction, and in many cases using multielectrode arrays. Nevertheless, if the structure is $2 \mathrm{D}$, valuable information can be added if deployments perpendicular to the profile are included, particularly for archaeological prospecting, where the main objective is the delineation of buried structures rather than the electrical characterization of the media. Various authors calculated the theoretical responses of 2D structures, both for parallel and perpendicular deployments, and found that for arrays perpendicular to the profile, the responses are sensitive to lateral variations only when they are close or just over the inhomogeneities (Van Nostrand and Cook, 1955; Mundry, 1984; Schulz and Tezkan, 1988; Pous et al., 1996). Hence, these arrays can constrain the localization of the structures.

In a previous paper, we presented a method for 2D resistivity modeling as an alternative to finite element (FE) and finite difference (FD) techniques (Osella et al., 2000). This method is based on a Rayleigh-Fourier approach, and is especially adequate to model layered structures with smooth 
irregular boundaries, which can be difficult to model with FE or FD codes, because of the complexity of the grids required to consider this particular kind of models. In that work, we only considered the case of parallel deployments. The validity of the method was verified by comparing the results with the ones obtained using a FE technique by Pous et al. (1996), and its ability to model actual data was tested by applying it to image an alluvial aquifer in Sierras Pam-

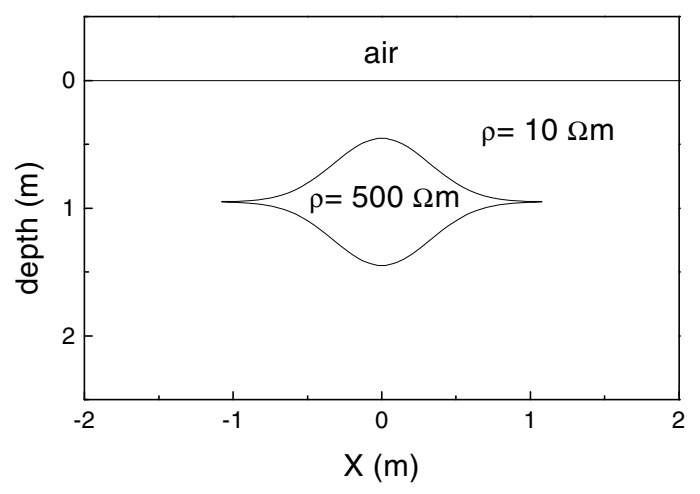

Fig. 1. Geoelectrical model to simulate a shallow 2D resistive structure. peanas region (Osella et al., 1999). In the present work, we modify this method to allow electrodes to be located in any position, and to include the case of three-dimensional (3D) structures. For 2D structures, we can obtain two apparent resistivity curves at each site along a profile perpendicular to the strike, one for electrodes deployed parallel to the profile (the usual way) and the other perpendicular to it. With these results, we can build up two pseudosections, in a similar way as is done for the transverse electric (TE) and transverse magnetic (TM) modes in magnetotelluric (MT) and audiomagnetotelluric (AMT) data.

Using this formulation we numerically simulate the response of simple 2D models. First we consider two shallow structures, a resistive body embedded in a conductive medium and a conductor in a resistive host, and we analyze the advantages in using both parallel and perpendicular pseudosections to model the structure from the synthetic data. Then, we consider two deeper bodies (again, one resistive and other conductive) and compare their geoelectric results with the corresponding AMT responses. Finally, we carried out a geoelectrical survey to image a known shallow buried structure, in order to show the goodness of the model fit obtained using this methodology.

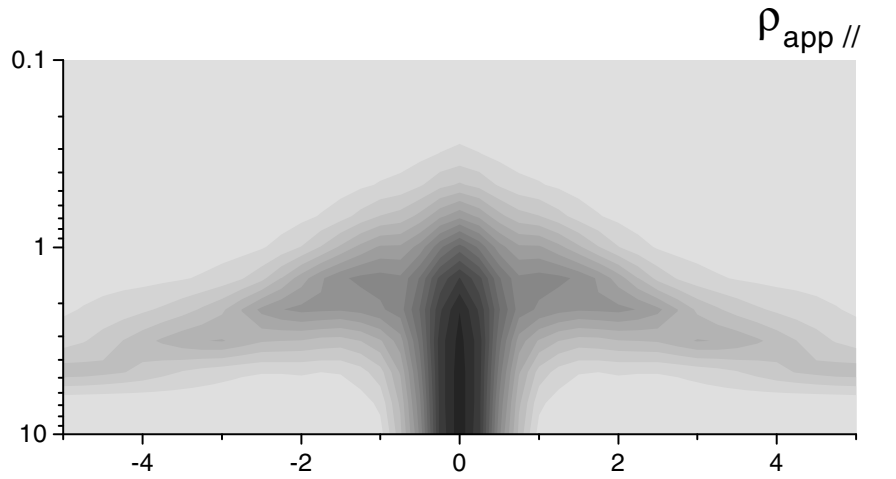

$\rho(\Omega m)$

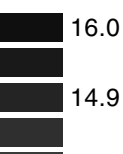

(a)

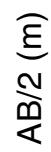

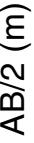

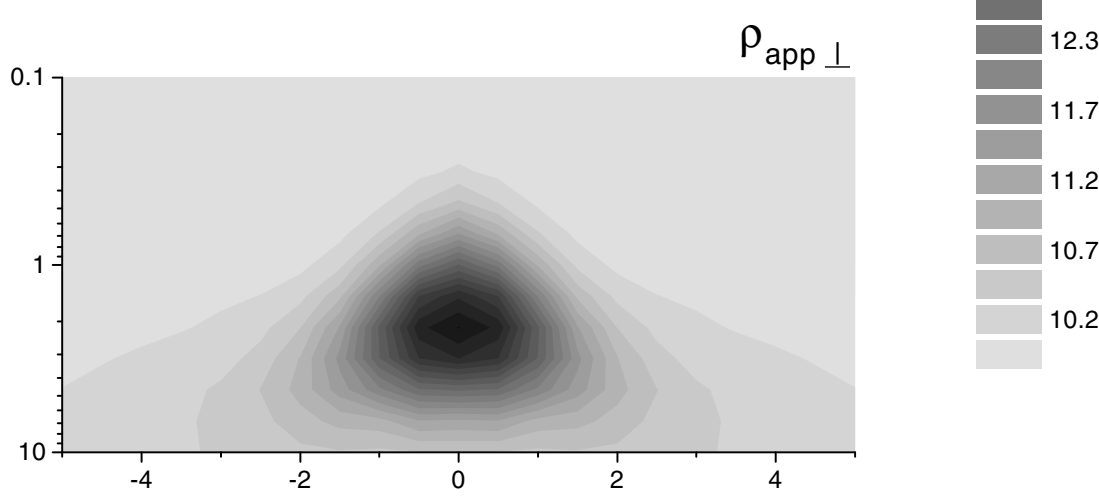

$X(m)$

Fig. 2. Pseudosections corresponding to deployments (a) parallel $\left(\rho_{\text {app } / /}\right)$ and (b) perpendicular $\left(\rho_{\text {app } \perp)}\right)$ to the profile. The profile is assumed to be perpendicular to the strike direction. 


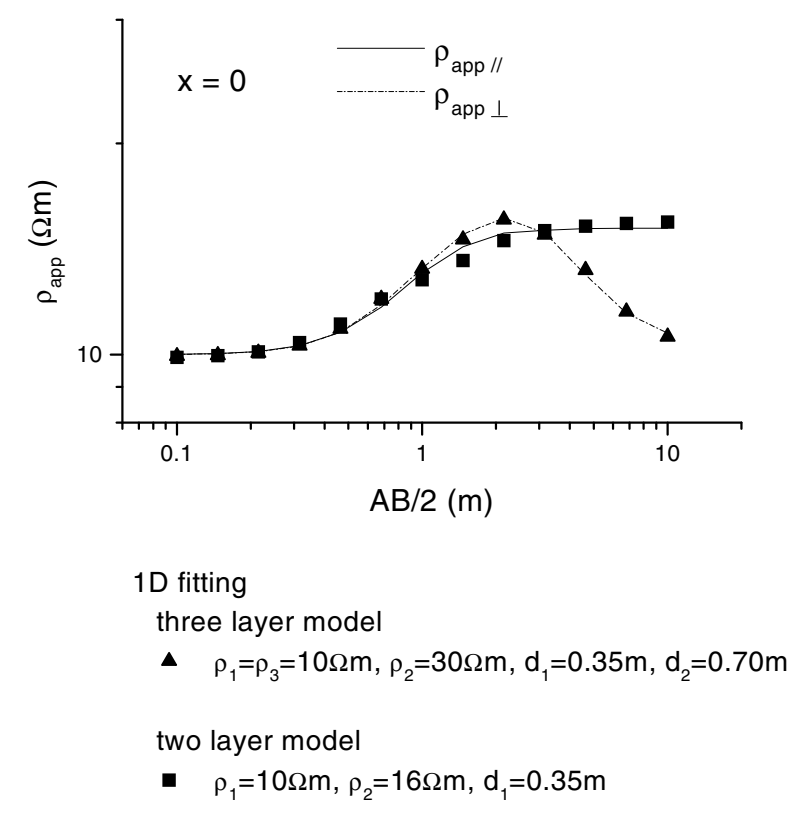

Fig. 3. 1D model responses of apparent resistivity corresponding to the center of the structure. The $\rho_{a p p / /}$ component was fitted by a two-layer model while the $\rho_{a p p \perp}$ component by a three-layer model. In both cases, layer 2 represents the resistive body and layers 1 and 3 the host medium.

\section{Theoretical Modeling}

In a previous paper, Osella et al. (2000) presented the method applied to 2D structures with a fixed electrode configuration, deployed along the direction of the profile. In the present work this formulation is extended to describe the electrical response for any array configuration and 3D structures. The medium is assumed to be $N$-layered, with boundaries given by functions $z=S^{(n)}(x, y), 0 \leq n \leq N-1$. Each layer, $n$, is isotropic and homogeneous, with electrical conductivity $\sigma^{(n)}$ and resistivity $\rho^{(n)}$. For $n=0, \sigma^{(0)} \rightarrow 0$; the free surface, given by $n=0$, can include topography, although in this case it is assumed to be horizontal $\left(S^{(0)}=0\right.$, $\forall(x, y))$. Current and potential electrodes, $A$ and $B$ and $C$ and $D$, respectively, are located on this surface, at $\left(x_{A}, y_{A}\right)$, $\left(x_{B}, y_{B}\right),\left(x_{C}, y_{C}\right)$ and $\left(x_{D}, y_{D}\right)$, respectively.

Inside each layer, Laplace's equation holds:

$$
\nabla^{2} \Phi^{(n)}(x, y, z)=0
$$

and the current density, $\vec{J}^{(n)}$, satisfies:

$$
\vec{J}^{(n)}=-\sigma^{(n)} \vec{\nabla} \Phi^{(n)} .
$$

In order to apply the Rayleigh-Fourier expansions, the boundaries are assumed to be periodic both in $x$ and $y$ coordinates, with periodicity $\lambda$. The area of application is limited to a short region (compared to $\lambda$ ) and centered at

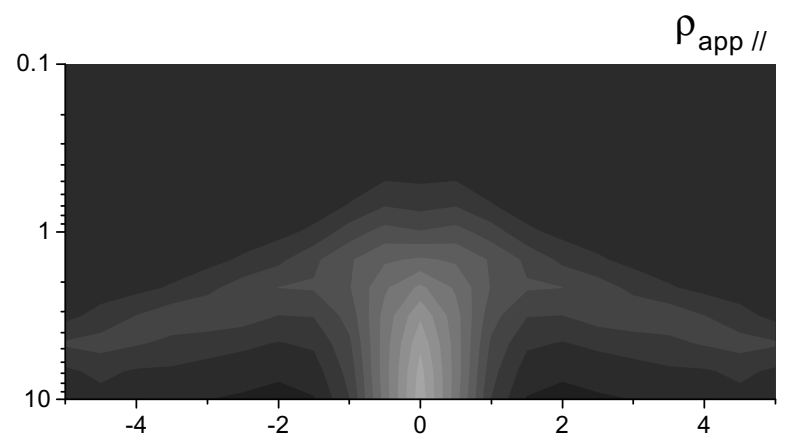

$\stackrel{\aleph}{\longleftarrow}$

(a)

$\rho(\Omega \mathrm{m})$

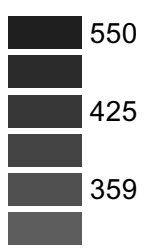

302
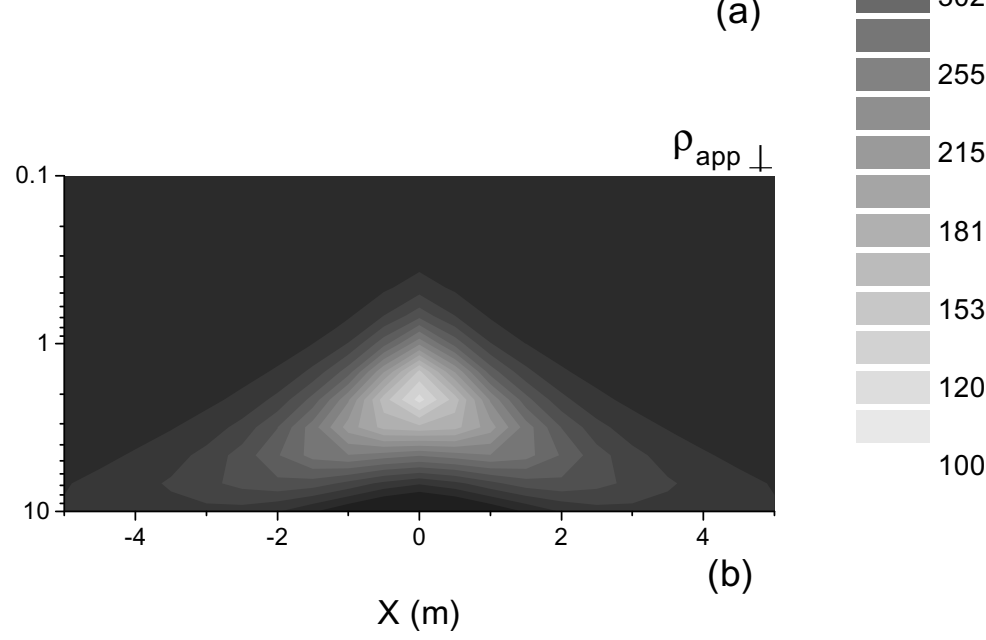

215

153

120

100

Fig. 4. Pseudosections corresponding to deployments (a) parallel $\left(\rho_{a p p / /}\right)$ and (b) perpendicular $\left(\rho_{\text {app } \perp)}\right)$ to the profile, for the structure shown in Fig. 1, but with $\rho_{1}=\rho_{3}=500 \Omega \mathrm{m}$ and $\rho_{2}=10 \Omega \mathrm{m}$. The profile is assumed to be perpendicular to the strike direction. 
$(x, y)=(\lambda / 4, \lambda / 4)$. For simplicity and without loosing generality, $z=S^{(n)}(x, y)$ is taken an even function of $x$ and $y$, for all $n$.

For $n=1$, the source terms of the potential are given by:

$$
\begin{aligned}
\Phi_{S}^{(1)}(x, y, z)= & \frac{I}{2 \pi \sigma^{(1)}} \\
& \times\left\{\frac{1}{\left[\left(x-x_{A}\right)^{2}+\left(y-y_{A}\right)^{2}+z^{2}\right]^{1 / 2}}\right. \\
& \left.-\frac{1}{\left[\left(x-x_{B}\right)^{2}+\left(y-y_{B}\right)^{2}+z^{2}\right]^{1 / 2}}\right\} \\
& +\varphi(x, y, z),
\end{aligned}
$$

with $I$ the injected current and $\varphi$ the potential of the sources located out of the area of application. The complete expresion for the potential for $n=1$ is:

$$
\begin{aligned}
\Phi^{(1)}(x, y, z)= & \Phi_{S}^{(1)}(x, y, z)+\sum_{l, m \geq 0}\left[A_{l m}^{(1)} \exp \left(k_{l m} z\right)\right. \\
& \left.+B_{l m}^{(1)} \exp \left(-k_{l m} z\right)\right] \\
& \times \cos \left(k_{l} x\right) \cos \left(k_{m} y\right)
\end{aligned}
$$

with $k_{l}=2 \pi l / \lambda, k_{m}=2 \pi m / \lambda$ and $k_{l m}=\left[k_{l m}^{2}+k_{l m}^{2}\right]^{1 / 2}$. For the following layers $(n \geq 2)$, the potential is written as:

$$
\begin{aligned}
\Phi^{(n)}(x, y, z)= & \sum_{l, m \geq 0}\left[A_{l m}^{(n)} \exp \left(k_{l m} z\right)+B_{l m}^{(n)} \exp \left(-k_{l m} z\right)\right] \\
& \times \cos \left(k_{l} x\right) \cos \left(k_{m} y\right) .
\end{aligned}
$$

The application of boundary conditions and subsequent projection onto sine and cosine basis lead to the following expressions (Appendix):

$$
\begin{gathered}
\sum_{l, m}\left[2 A_{l m}^{(1)} E_{l^{\prime} m^{\prime} l m}+V_{l m} D_{l^{\prime} m^{\prime} l m}\right. \\
\left.-A_{l m}^{(2)} F_{l^{\prime} m^{\prime} l m}^{(1)}-B_{l m}^{(2)} G_{l^{\prime} m^{\prime} l m}^{(1)}\right]=0, \\
\sum_{l, m}\left[2 \sigma^{(1)} A_{l m}^{(1)} O_{l^{\prime} m^{\prime} l m}+\sigma^{(1)} W_{l m} D_{l^{\prime} m^{\prime} l m} / \lambda\right. \\
\left.-\sigma^{(2)} A_{l m}^{(2)} P_{l^{\prime} m^{\prime} l m}^{(1)}-\sigma^{(2)} B_{l m}^{(2)} Q_{l^{\prime} m^{\prime} l m}^{(1)}\right]=0, \\
\sum_{l, m}\left[\left(A_{l m}^{(j)}-A_{l m}^{(j+1)}\right) F_{l^{\prime} m^{\prime} l m}^{(j)}\right. \\
\left.+\left(B_{l m}^{(j)}-B_{l m}^{(j+1)}\right) G_{l^{\prime} m^{\prime} l m}^{(j)}\right]=0, \\
\sum_{l, m}\left[\left(\sigma^{(j)} A_{l m}^{(j)}-\sigma^{(j+1)} A_{l m}^{(j+1)}\right) P_{l^{\prime} m^{\prime} l m}^{(j)}\right. \\
\left.+\left(\sigma^{(j)} B_{l m}^{(j)}-\sigma^{(j+1)} B_{l m}^{(j+1)}\right) Q_{l^{\prime} m^{\prime} l m}^{(j)}\right]=0, \\
A_{l m}^{(1)}=B_{l m}^{(1)} . \\
A_{l m}^{(N)}=0, \quad \forall l, m,
\end{gathered}
$$

where

$$
\begin{aligned}
& O_{l^{\prime} m^{\prime} l m}=k_{l m} H_{l^{\prime} m^{\prime} l m}+k_{l} I_{l^{\prime} m^{\prime} l m}+k_{m} J_{l^{\prime} m^{\prime} l m}, \\
& P_{l^{\prime} m^{\prime} l m}^{(j)}=k_{l m} F_{l^{\prime} m^{\prime} l m}^{(j)}+k_{l} K_{l^{\prime} m^{\prime} l m}^{(j)}+k_{m} M_{l^{\prime} m^{\prime} l m}^{(j)}, \\
& Q_{l^{\prime} m^{\prime} l m}^{(j)}=-k_{l m} G_{l^{\prime} m^{\prime} l m}^{(j)}+k_{l} L_{l^{\prime} m^{\prime} l m}^{(j)}+k_{m} N_{l^{\prime} m^{\prime} l m}^{(j)},
\end{aligned}
$$

$$
\begin{aligned}
D_{l^{\prime} m^{\prime} l m}= & \int_{-\lambda / 2}^{\lambda / 2} \int_{-\lambda / 2}^{\lambda / 2} \cos \left(k_{l} x\right) \cos \left(k_{m} y\right) \cos \left(k_{l^{\prime}} x\right) \\
& \times \cos \left(k_{m^{\prime}} y\right) d x d y=\frac{\lambda^{2} \delta_{l l^{\prime}} \delta_{m m^{\prime}}}{\left(2-\delta_{l 0}\right)\left(2-\delta_{m 0}\right)}, \\
E_{l^{\prime} m^{\prime} l m}= & \int_{-\lambda / 2}^{\lambda / 2} \int_{-\lambda / 2}^{\lambda / 2} \operatorname{ch}\left(k_{l m} S^{(1)}(x, y)\right) \cos \left(k_{l} x\right) \cos \left(k_{m} y\right) \\
& \times \cos \left(k_{l^{\prime}} x\right) \cos \left(k_{m^{\prime}} y\right) d x d y, \\
F_{l^{\prime} m^{\prime} l m}^{(j)}= & \int_{-\lambda / 2}^{\lambda / 2} \int_{-\lambda / 2}^{\lambda / 2} \exp \left(k_{l m} S^{(j)}(x, y)\right) \cos \left(k_{l} x\right) \\
& \times \cos \left(k_{m} y\right) \cos \left(k_{l^{\prime}} x\right) \cos \left(k_{m^{\prime}} y\right) d x d y, \\
G_{l^{\prime} m^{\prime} l m}^{(j)}= & \int_{-\lambda / 2}^{\lambda / 2} \int_{-\lambda / 2}^{\lambda / 2} \exp \left(-k_{l m} S^{(j)}(x, y)\right) \cos \left(k_{l} x\right) \\
& \times \cos \left(k_{m} y\right) \cos \left(k_{l^{\prime}} x\right) \cos \left(k_{m^{\prime}} y\right) d x d y, \\
H_{l^{\prime} m^{\prime} l m}= & \int_{-\lambda / 2}^{\lambda / 2} \int_{-\lambda / 2}^{\lambda / 2} \operatorname{sh}\left(k_{l m} S^{(1)}(x, y)\right) \cos \left(k_{l} x\right) \cos \left(k_{m} y\right) \\
& \times \cos \left(k_{l^{\prime}} x\right) \cos \left(k_{m^{\prime}} y\right) d x d y, \\
I_{l^{\prime} m^{\prime} l m}= & \int_{-\lambda / 2}^{\lambda / 2} \int_{-\lambda / 2}^{\lambda / 2} \frac{\partial S^{(1)}(x, y)}{\partial x} \operatorname{ch}\left(k_{l m} S^{(1)}(x, y)\right) \\
& \times \sin \left(k_{l} x\right) \cos \left(k_{l} y\right) \cos \left(k_{l} x\right) \cos \left(k_{l,} y\right) d x d y, \\
&
\end{aligned}
$$$$
\times \sin \left(k_{l} x\right) \cos \left(k_{m} y\right) \cos \left(k_{l^{\prime}} x\right) \cos \left(k_{m^{\prime}} y\right) d x d y,
$$

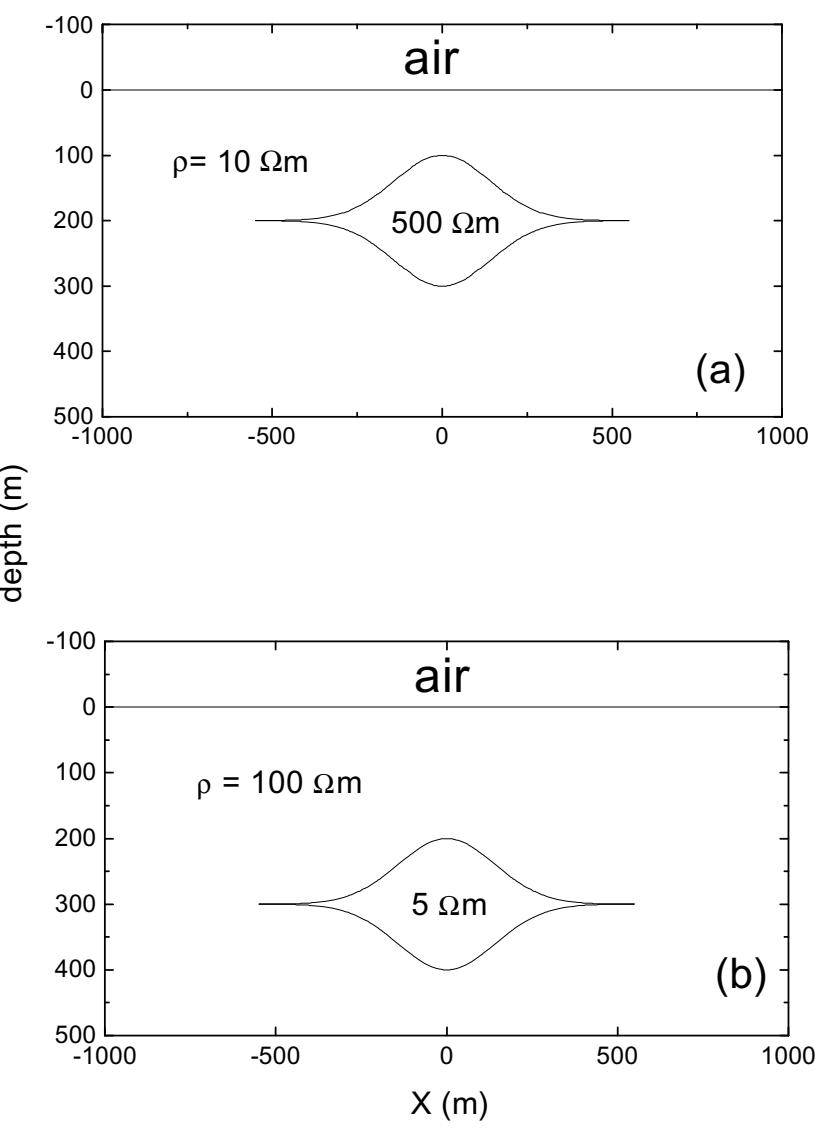

Fig. 5. Electrical models to simulate a deep 2D (a) resistive and (b) conductive body. 

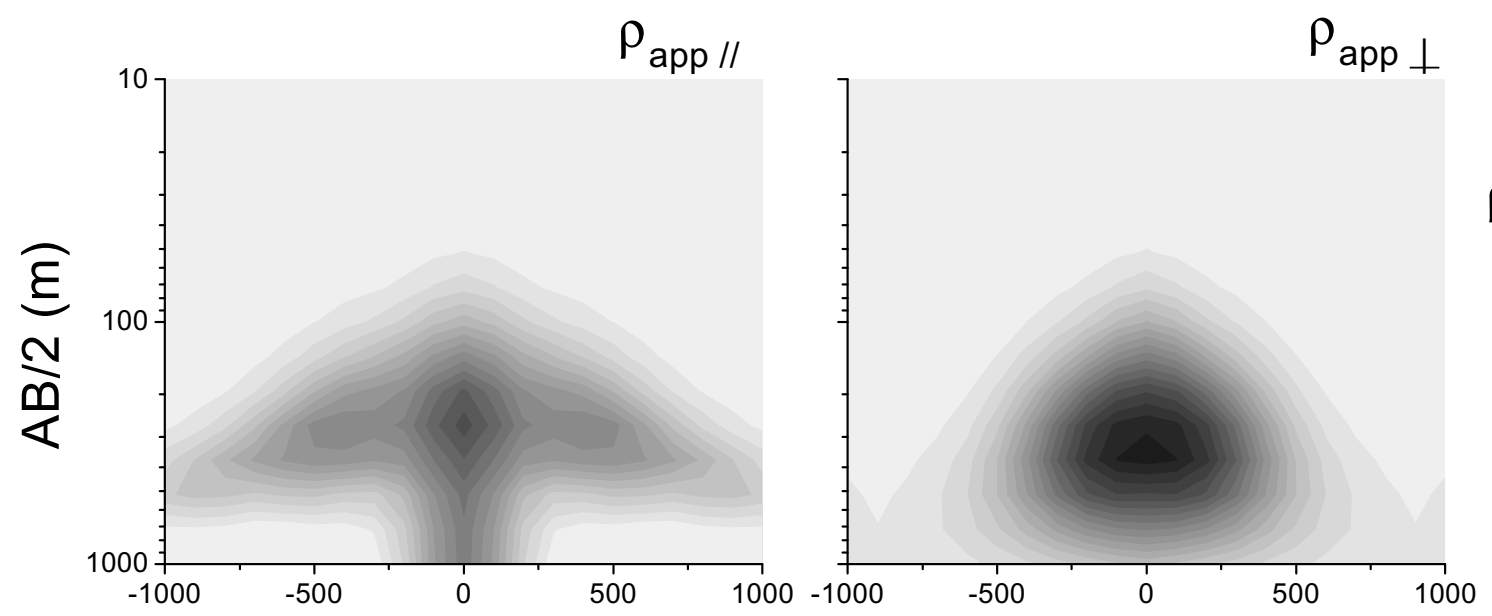

$\rho(\Omega \mathrm{m})$
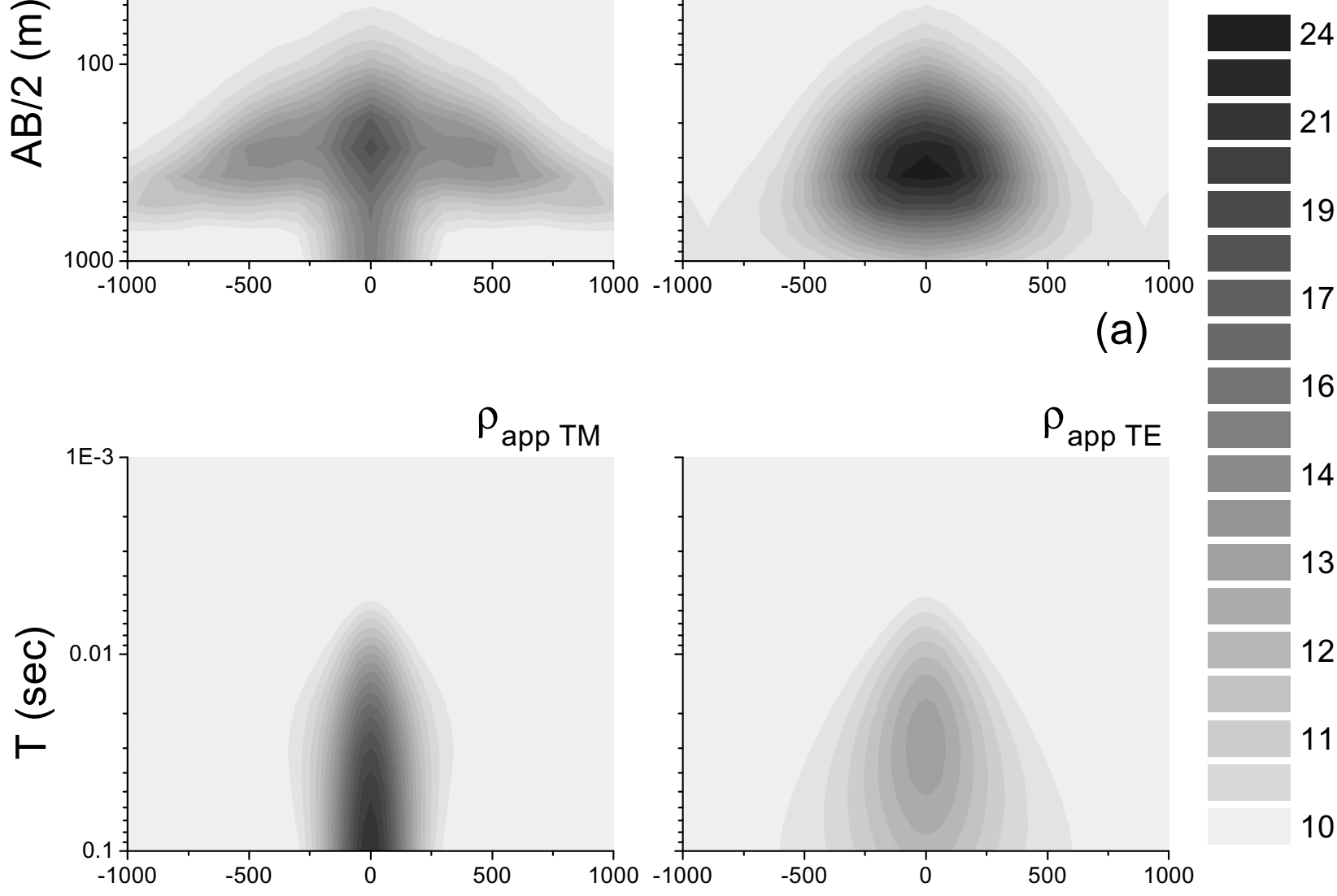

16

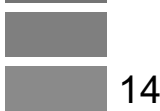

14

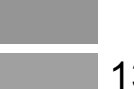

13

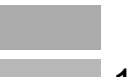

(b)

\section{$X(m)$}

Fig. 6. Pseudosections of the electrical response due to the model shown in Fig. 5(a). (a) The geoelectrical responses corresponding to the parallel ( $\rho_{a p p / /)}$ and perpendicular $\left(\rho_{\text {app } \perp}\right)$ deployments. (b) AMT responses for transverse magnetic $\left(\rho_{\text {app TM }}\right)$ and transverse electric $\left(\rho_{\text {appTE }}\right)$ modes.

$$
\begin{aligned}
J_{l^{\prime} m^{\prime} l m}= & \int_{-\lambda / 2}^{\lambda / 2} \int_{-\lambda / 2}^{\lambda / 2} \frac{\partial S^{(1)}(x, y)}{\partial y} \operatorname{ch}\left(k_{l m} S^{(1)}(x, y)\right) \\
& \times \cos \left(k_{l} x\right) \sin \left(k_{m} y\right) \cos \left(k_{l^{\prime}} x\right) \cos \left(k_{m^{\prime}} y\right) d x d y,
\end{aligned}
$$

$$
K_{l^{\prime} m^{\prime} l m}^{(j)}=\int_{-\lambda / 2}^{\lambda / 2} \int_{-\lambda / 2}^{\lambda / 2} \frac{\partial S^{(j)}(x, y)}{\partial x} \exp \left(k_{l m} S^{(j)}(x, y)\right)
$$

$\times \sin \left(k_{l} x\right) \cos \left(k_{m} y\right) \cos \left(k_{l^{\prime}} x\right) \cos \left(k_{m^{\prime}} y\right) d x d y$,

$$
\begin{aligned}
L_{l^{\prime} m^{\prime} l m}^{(j)}= & \int_{-\lambda / 2}^{\lambda / 2} \int_{-\lambda / 2}^{\lambda / 2} \frac{\partial S^{(j)}(x, y)}{\partial x} \exp \left(-k_{l m} S^{(j)}(x, y)\right) \\
& \times \sin \left(k_{l} x\right) \cos \left(k_{m} y\right) \cos \left(k_{l^{\prime}} x\right) \cos \left(k_{m^{\prime}} y\right) d x d y,
\end{aligned}
$$

$$
\begin{aligned}
M_{l^{\prime} m^{\prime} l m}^{(j)}= & \int_{-\lambda / 2}^{\lambda / 2} \int_{-\lambda / 2}^{\lambda / 2} \frac{\partial S^{(j)}(x, y)}{\partial y} \exp \left(k_{l m} S^{(j)}(x, y)\right) \\
& \times \cos \left(k_{l} x\right) \sin \left(k_{m} y\right) \cos \left(k_{l^{\prime}} x\right) \cos \left(k_{m^{\prime}} y\right) d x d y,
\end{aligned}
$$

$$
\begin{aligned}
N_{l^{\prime} m^{\prime} l m}^{(j)}= & \int_{-\lambda / 2}^{\lambda / 2} \int_{-\lambda / 2}^{\lambda / 2} \frac{\partial S^{(j)}(x, y)}{\partial y} \exp \left(-k_{l m} S^{(j)}(x, y)\right) \\
& \times \cos \left(k_{l} x\right) \sin \left(k_{m} y\right) \cos \left(k_{l^{\prime}} x\right) \cos \left(k_{m^{\prime}} y\right) d x d y,
\end{aligned}
$$

$$
\begin{aligned}
V_{l m}= & \frac{\left(2-\delta_{l 0}\right)\left(2-\delta_{m 0}\right)}{\lambda^{2}} \\
& \times \int_{-\lambda / 2}^{\lambda / 2} \int_{-\lambda / 2}^{\lambda / 2} \Phi_{F}^{(1)}\left(x, y, S^{(1)}(x, y)\right) \\
& \times \cos \left(k_{l} x\right) \cos \left(k_{m} y\right) d x d y,
\end{aligned}
$$




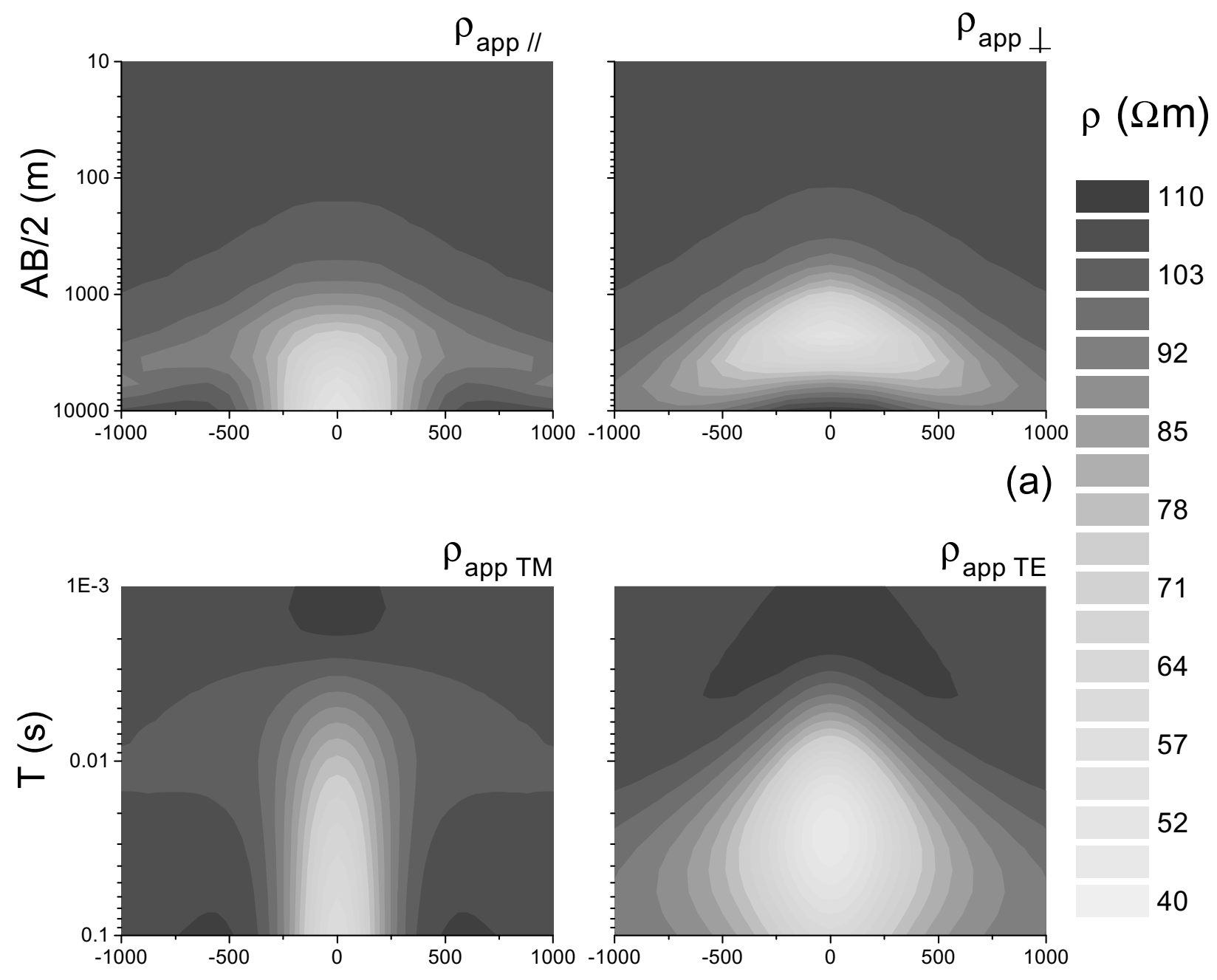

(b)

\section{$X(m)$}

Fig. 7. Pseudosections of the electrical response due to the model shown in Fig. 5(b) . (a) The geoelectrical responses corresponding to the parallel $\left(\rho_{\text {app } / /}\right)$ and perpendicular $\left(\rho_{\text {app } \perp}\right)$ deployments. (b) AMT responses for transverse magnetic $\left(\rho_{\text {appTM }}\right)$ and transverse electric $\left(\rho_{\text {appTE }}\right)$ modes.

$$
\begin{aligned}
W_{l m}= & \frac{\left(2-\delta_{l 0}\right)\left(2-\delta_{m 0}\right)}{\lambda} \\
& \times \int_{-\lambda / 2}^{\lambda / 2} \int_{-\lambda / 2}^{\lambda / 2}\left[\frac{\partial \Phi_{F}^{(1)}}{\partial z}\left(x, y, S^{(1)}(x, y)\right)\right. \\
& -\frac{\partial S^{(1)}(x, y)}{\partial x} \frac{\partial \Phi_{F}^{(1)}}{\partial x}\left(x, y, S^{(1)}(x, y)\right) \\
& \left.-\frac{\partial S^{(1)}(x, y)}{\partial y} \frac{\partial \Phi_{F}^{(1)}}{\partial y}\left(x, y, S^{(1)}(x, y)\right)\right] \\
& \quad \times \cos \left(k_{l} x\right) \cos \left(k_{m} y\right) d x d y .
\end{aligned}
$$

Up to this point no assumptions have been done on the dimensionality of the structure, so it can be applied also to $3 \mathrm{D}$ modeling. But if a symmetry axis exists, Eqs. (15) to (27) are largely simplified. Under this assumption, for $S^{(j)}=S^{(j)}(x), \forall j, J_{l^{\prime} m^{\prime} l m}^{(j)}=M_{l^{\prime} m^{\prime} l m}^{(j)}=N_{l^{\prime} m^{\prime} l m}^{(j)}=0$, while the rest of the expressions include only simple integrals.

The unknowns in the system of equations given by (6)-(9) are the Fourier coefficients $A_{l m}^{(j)}$ and $B_{l m}^{(j)}$. Rayleigh's scattering theory actually constitutes an approximation because some multiple reflections are not accounted. Nevertheless, when this approximation is valid, the series are convergent and these coefficients are negligible for $l, m>L$. That is to say that for a given $j$, the number of unknowns to be calculated from Eqs. (6) to (9) is reduced to $2(L+1)^{2}$. Taking into account (10) and (11), it is clear that the number of independent equations to be solved from $(6)-(9)$ is $(L+1)^{2}$, for $j=1, N$, and $2(L+1)^{2}$, for $j \neq 1, N$. For $l^{\prime}=m^{\prime}=0$, Eqs. (7) and (9) do not depend either on $A_{00}^{(j)}$ or $B_{00}^{(j)}$, hence we write:

$$
2 \sigma^{(1)} A_{00}^{(1)}+\sigma^{(1)} W_{00}-\sigma^{(2)} A_{00}^{(2)}+\sigma^{(2)} B_{00}^{(2)}=0,
$$




$$
\begin{aligned}
\left(\sigma^{(j)} A_{00}^{(j)}\right. & \left.-\sigma^{(j+1)} A_{00}^{(j+1)}\right) \\
& -\left(\sigma^{(j)} B_{00}^{(j)}-\sigma^{(j+1)} B_{00}^{(j+1)}\right)=0,
\end{aligned}
$$

to obtain an independent system. Equations (6)-(9) and (28)-(29) can be expressed in matricial form; solving this system together with Eqs. (10) and (11) for $\mathbf{A}^{(1)}$, the following is obtained:

$$
\mathbf{A}^{(1)}=-\mathbf{A}_{V} \cdot \mathbf{V}+\mathbf{A}_{W} \cdot \mathbf{W}
$$

where

$$
\begin{aligned}
\mathbf{A}_{V}= & \frac{1}{2}\left[\mathbf{E}-\frac{\sigma^{(1)}}{\sigma^{(2)}} \mathbf{S}^{(1)} \cdot\left(\mathbf{T}^{(1)}\right)^{-1} \cdot \mathbf{O}\right]^{-1} \cdot \mathbf{D} \\
\mathbf{A}_{W}= & \frac{1}{2 \lambda}\left[\frac{\sigma^{(2)}}{\sigma^{(1)}} \mathbf{T}^{(1)} \cdot\left(\mathbf{S}^{(1)}\right)^{-1} \cdot \mathbf{E}-\mathbf{O}\right]^{-1} \cdot \mathbf{D}, \\
\mathbf{S}^{(j)}= & \mathbf{F}^{(j)} \cdot \mathbf{R}^{(j+1)}+\mathbf{G}^{(j)} \\
\mathbf{T}^{(j)}= & \mathbf{P}^{(j)} \cdot \mathbf{R}^{(j+1)}+\mathbf{Q}^{(j)} \cdot \\
\mathbf{R}^{(j)}= & {\left[\left(\mathbf{S}^{(j)}\right)^{-1} \cdot \mathbf{F}^{(j)}-\frac{\sigma^{(j)}}{\sigma^{(j+1)}}\left(\mathbf{T}^{(j)}\right)^{-1} \cdot \mathbf{P}^{(j)}\right]^{-1} } \\
& \cdot\left[-\left(\mathbf{S}^{(j)}\right)^{-1} \cdot \mathbf{G}^{(j)}+\frac{\sigma^{(j)}}{\sigma^{(j+1)}}\left(\mathbf{T}^{(j)}\right)^{-1} \cdot \mathbf{Q}^{(j)}\right] \\
\mathbf{R}^{(N)}= & 0
\end{aligned}
$$

From Eq. (30) the Fourier coefficients, $A_{l m}^{(1)}$ for the potential $\Phi^{(1)}(x, y, z)$, corresponding to the shallow layer (Eq. (4)), can be calculated. It can be noted that in Eq. (30) the first factors, $A_{v}$ and $A_{w}$, depend only on the conductivities and geometries of the $N$ interfaces. In contrast, the second factors, $V$ and $W$, depend on the source distribution and the characteristics of the first layer. In this sense, $\mathbf{A}_{V}$ and $\mathbf{A}_{W}$ can be interpreted as the effective response of the structure to a given source distribution.

A self-consistency criterion for the determination of the validity of the Rayleigh approach in each particular case has been proposed in the work by Osella et al. (2000) that is also valid for this generalized method. As Rayleigh solutions are an approximation, there are residual discontinuities of $\Phi$ and the normal component of $\vec{J}$ at layer interfaces. When the approximation is valid, the root mean square value of these residuals can be reduced to a level below a few per cent, by increasing the number of scattering orders, $L$. This is because the series are convergent. When the approximation is not valid, the residual discontinuities remain large, and the series exhibit an oscillatory behavior or converge for small values of $L$ and then diverge as $L$ increases.

\section{Numerical Simulation}

We propose the 2D model shown in Fig. 1 to simulate a buried resistive structure. We calculate the theoretical responses along a profile perpendicular to the strike in the Schlumberger configuration. At each site along the profile, two apparent resistivity curves are calculated, $\rho_{a p p / / \text {, }}$ with deployments parallel to the profile and $\rho_{a p p \perp}$, with electrodes deployed perpendicular to the profile, respectively. The resulting pseudosections for $\rho_{a p p / /}$, (Fig. 2(a)) and $\rho_{a p p \perp}$, (Fig. 2(b)), show different behaviors.
At sites close to the center of the structure $\rho_{a p p \perp}$ approaches the half space resistivity $10 \Omega \mathrm{m}$, as $A B / 2$ increases, while $\rho_{\text {app } / /}$ does not recover this value even for apertures $A B / 2$ larger than several times the characteristic dimension of the body. These results can also be seen from the apparent resistivity curves, obtained at the center of the structure for both configurations in Fig. 3. If a 1D modeling is performed, the $\rho_{a p p / /}$ curve can be fit with a two-layer model, while the $\rho_{a p p \perp}$ component fits a three-layer model. The location of the resistive layer is close to the actual position of the structure, although the resistivity, as expected, is underestimated.

The behavior far from the structure is also different for both cases. The effect of the resistive body is detected on the parallel configuration at distances three to four times its characteristic width. On the other hand, the $\rho_{\text {app } \perp}$ component shows a localized distortion both in width and depth, and rapidly recovers the half-space behavior. This result implies that the $\rho_{a p p \perp}$ component should be used for imaging the embedding medium, because $\rho_{a p p / /}$ gives a distorted result even far from the structure.

We now consider the same structure as in Fig. 1, but assuming a body of $\rho=10 \Omega \mathrm{m}$ embedded in a medium of $\rho=500 \Omega \mathrm{m}$. The response, shown in Fig. 4, presents similar features to that of Fig. 2. It is interesting to note that in this case the effect of the conducting body is detected,
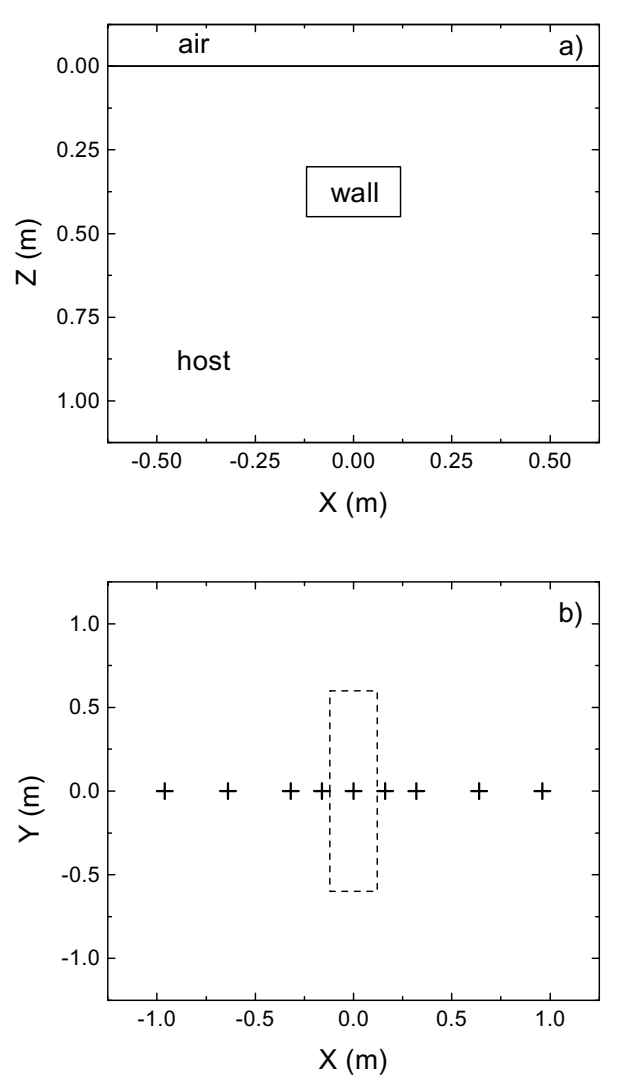

Fig. 8. a) Scheme of the structure used to test our method. This structure is a part of a wall of an ancient fort, which was detected from preliminary excavations made in a small zone of an archaeological site in South Argentina. b) Plan view showing the studied profile. Parallel and perpendicular measurements were carried out at each sounding site using the Schlumberger configuration. 


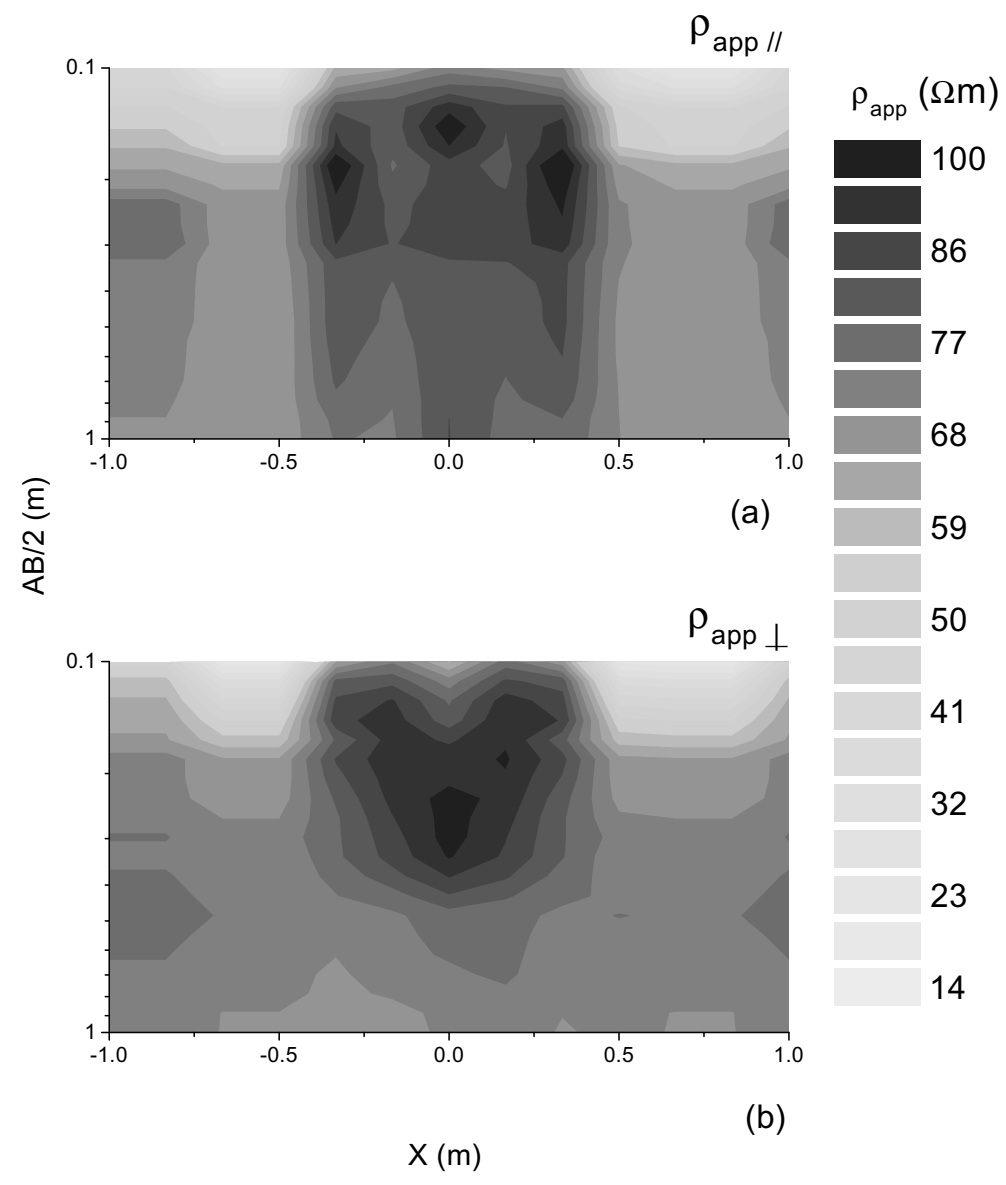

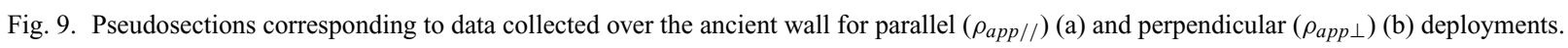

especially for the $\rho_{\text {app // }}$ curve, at larger values of $A B$.

Next we analyze the behavior of two deeper structures: a resistive body in a conductive medium shown in Fig. 5(a), and a conductive body embedded in a resistive medium shown in Fig. 5(b). The AMT responses were also calculated for these models. The AMT apparent resistivities corresponding to the $T M$ and $T E$ modes, $\rho_{T M}$ and $\rho_{T E}$ respectively, were obtained applying a Rayleigh-Fourier method previously implemented by Osella and Martinelli (1993). In Fig. 6, the results are shown for the case of the resistive body, while in Fig. 7 the results are shown for the conductive one.

Similar characteristics are observed between the geoelectrical responses corresponding to the shallow (Fig. 2) and to the deep (Fig. 6(a)) resistive body. Again, far from the structure, the $\rho_{a p p / /}$ component is more sensitive to the $2 \mathrm{D}$ body, while the $\rho_{a p p \perp}$ component more rapidly recovers the $1 \mathrm{D}$ response. Close to the center of the body, $\rho_{a p p \perp}$ gives a constrain for the depth of the lower boundary of the target. Similarities with the behavior of $\rho_{T M}$ and $\rho_{T E}$ can be clearly observed by comparison to Fig. 6(b). The $T M$ resistivity is more distorted close to the center of the anomaly, but more rapidly recovers the host response. The $T E$ resistivity gives a mean approximation of the structure. The anomaly in the response is wider than the corresponding to the $T M$ mode, and the lower boundary of the body is not clearly constrained.

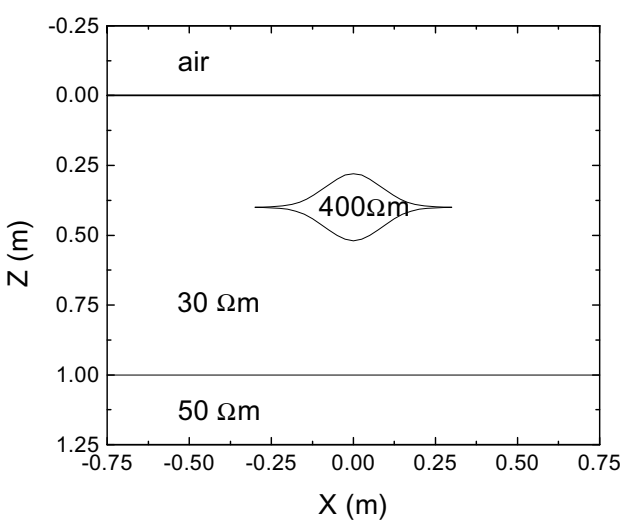

Fig. 10. Model of the ancient wall obtained after applying a trial and error, least squares method to fit the data.

If we consider the structure shown in Fig. 5(b), a conductive body embedded in a resistive medium, both the geoelectrical and AMT responses follow a similar pattern, as can be observed in Figs. 7(a) and (b), respectively. The most relevant feature is that large $A B / 2$ distances are needed to detect the effect of the conducting structure on the geoelectrical responses compared to a resistive structure. For the conductive target at depth, the required electrode spacings should be larger than a kilometer. Hence, this method is 

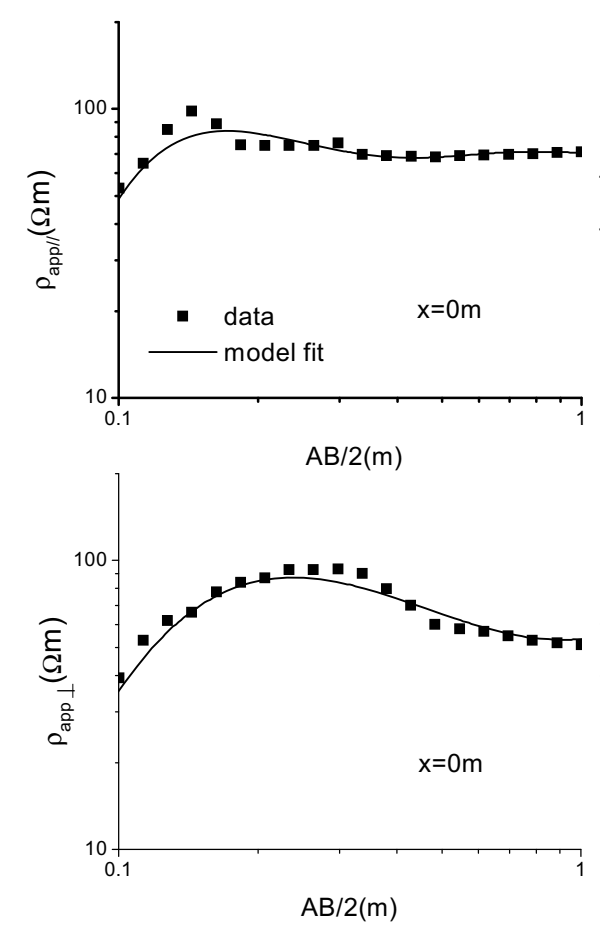

Fig. 11. Measured apparent resistivity curves $\rho_{a p p / /}$ and $\rho_{a p p \perp}$ obtained from parallel and perpendicular deployments, respectively, at some representative sites, together with the corresponding model responses. The misfit of the model fit is approximately $20 \%$. more appropriate to image resistive rather than conductive structures, because such distances make the application impractical.

\section{Application}

Recently, preliminary excavations have been made in a small zone of an archaeological site in South Argentina. From these excavations, a part of a wall of an ancient fort was detected, buried close to the surface (almost outcropping). A scheme of the structure is shown in Fig. 8(a). We used it as a target to test our method. We designed a nine sounding profile using the Schlumberger configuration, with parallel and perpendicular arrays deployed at each site (Fig. 8(b)). A HP E3612A DC power supply was used to inject current and measurements were collected using a data acquisition system that allows readings with a precision of $0.33 \mu \mathrm{V}$. The electrodes were stainless-steel rods $25 \mathrm{~cm}$ long and $7 \mathrm{~mm}$ diameters, in order to guarantee the pointsource assumption, given the minimum separation between potential electrodes was about $3 \mathrm{~cm}$. Pseudosections for the $\rho_{a p p / /}$ and $\rho_{a p p \perp}$ components are shown in Fig. 9. It can be seen that the location of the anomalous body is clearly detected.

We apply a trial and error method to fit the data. As a starting model, we proposed a buried 2D structure with smooth contours, similar to the one shown in Fig. 1, embedded in a two layer medium. Values of the parameters that define the medium of $30 \Omega \mathrm{m}$ and $50 \Omega \mathrm{m}$ for the resistivities of the first and second layers, respectively, were obtained from a $1 \mathrm{D}$ inversion of the $\rho_{a p p \perp}$ responses away from the anomalous zone. We then varied the geometrical parameters applying a least square method. The best result was obtained for the model shown in Fig. 10, which is in close agreement with the actual structure. The measured apparent resistivity curves together with the model responses, at some representative sites, are shown in Fig. 11. The misfit of the model fit is approximately $20 \%$. If the fit is performed using only the parallel deployment, the results are slightly sensitive to the thickness of the body, and this parameter could not be estimated.

\section{Discussion}

During the modeling process, the method showed good convergence and proved friendly to use for the modeling of multi-layered structures with smooth boundaries, which can be difficult to solve applying finite differences or finite elements codes, due to the complexity of the grids needed to consider this particular kind of structures.

From the examples studied in the present work, we can conclude that considering both pseudosections should lead to a more accurate description of an anomalous zone. When a 2D anomaly is present, its effect on the perpendicular component is more focused, both in width and depth, than in the parallel component. Then, it is better to use the perpendicular component to model the host medium, because if the sounding is performed far from a $2 \mathrm{D}$ body, it should not be detected by this component.

It is well known that when inverting MT or AMT data using 2D codes, better resolution in the electrical imaging is obtained when both modes are considered. Due to the similar behavior of the geoelectric and AMT responses, it is expected that 2D imaging of geoelectric data including both arrays should lead to an optimization of the inversion process.

In addition, it is also known that the simultaneous 1D inversion of DC and AMT data improve the electrical descrip- 
tion of the medium (Jupp and Vozoff, 1975; Monteiro Santos et al., 1997). Hence, results of inversions made including both configurations could be further used in correlation with AMT 2D codes; it is clear that this kind of joint inversion should contribute to remove uncertainties allowing an improvement in the description of the actual structures.

Acknowledgments. This work was partially supported by Conicet and Universidad de Buenos Aires.

\section{Appendix A.}

As it is well known, $\vec{J}\left(J_{n}\right)=0$ at $S^{(0)}$, except at the injection points. The application of this condition to Eq. (4) yields to expression (10). In addition, $\Phi^{(n)}$ and $J_{n}$ are continuous at each boundary $S^{(j)}$, for $1 \leq j \leq N-1$. At $S^{(1)}$ gives:

$$
\begin{aligned}
\sum_{l, m}[ & \left.2 A_{l m}^{(1)} \operatorname{ch}\left(k_{l m} S^{(1)}(x, y)\right)+V_{l m}\right] \cos \left(k_{l} x\right) \cos \left(k_{m} y\right) \\
& =\sum_{l, m}\left[A_{l m}^{(2)} \exp \left(k_{l m} S^{(1)}(x, y)\right)\right. \\
& \left.+B_{l m}^{(2)} \exp \left(-k_{l m} S^{(1)}(x, y)\right)\right] \cos \left(k_{l} x\right) \cos \left(k_{m} y\right)
\end{aligned}
$$

$$
\begin{aligned}
\sigma^{(1)} \sum_{l, m} & \left\{\left[2 A_{l m}^{(1)} k_{l m} \operatorname{sh}\left(k_{l m} S^{(1)}(x, y)\right)+W_{l m} / \lambda\right]\right. \\
& \times \cos \left(k_{l} x\right) \cos \left(k_{m} y\right) \\
& +\left[2 A_{l m}^{(1)} \frac{\partial S^{(1)}(x, y)}{\partial x} k_{l} \operatorname{ch}\left(k_{l m} S^{(1)}(x, y)\right)\right] \\
& \times \sin \left(k_{l} x\right) \cos \left(k_{m} y\right) \\
& +\left[2 A_{l m}^{(1)} \frac{\partial S^{(1)}(x, y)}{\partial y} k_{m} \operatorname{ch}\left(k_{l m} S^{(1)}(x, y)\right)\right] \\
& \left.\times \cos \left(k_{l} x\right) \sin \left(k_{m} y\right)\right\} \\
= & \sigma^{(2)} \sum_{l, m}\left\{k _ { l m } \left[A_{l m}^{(2)} \exp \left(k_{l m} S^{(1)}(x, y)\right)\right.\right. \\
& \left.-B_{l m}^{(2)} \exp \left(-k_{l m} S^{(1)}(x, y)\right)\right] \cos \left(k_{l} x\right) \cos \left(k_{m} y\right) \\
& +k_{l} \frac{\partial S^{(1)}(x, y)}{\partial x}\left[A_{l m}^{(2)} \exp \left(k_{l m} S^{(1)}(x, y)\right)\right. \\
& \left.+B_{l m}^{(2)} \exp \left(-k_{l m} S^{(1)}(x, y)\right)\right] \sin \left(k_{l} x\right) \cos \left(k_{m} y\right) \\
& +k_{m} \frac{\partial S^{(1)}(x, y)}{\partial y}\left[A_{l m}^{(2)} \exp \left(k_{l m} S^{(1)}(x, y)\right)\right. \\
& \left.\left.+B_{l m}^{(2)} \exp \left(-k_{l m} S^{(1)}(x, y)\right)\right] \cos \left(k_{l} x\right) \sin \left(k_{m} y\right)\right\},
\end{aligned}
$$

In the first equation, $V_{l m}$ is the Fourier coefficient corresponding to the source potential $\left(\Phi_{S}^{(1)}\right)$ evaluated on $S^{(1)}(x, y)$; it has been defined in Eq. (25), while coefficient $W_{l m}$ has been defined in Eq. (27).

In a similar way, from the continuity of $\Phi^{(n)}$ and $J_{n}$ at
$S^{(j)}, 2 \leq j \leq(N-1)$, the following equations are obtained:

$$
\begin{aligned}
\sum_{l, m}[ & \left.A_{l m}^{(j)} \exp \left(k_{l m} S^{(j)}(x, y)\right)+B_{l m}^{(j)} \exp \left(-k_{l m} S^{(j)}(x, y)\right)\right] \\
& \times \cos \left(k_{l} x\right) \cos \left(k_{m} y\right) \\
& =\sum_{l, m}\left[A_{l m}^{(j+1)} \exp \left(k_{l m} S^{(j)}(x, y)\right)\right. \\
& \left.+B_{l m}^{(j+1)} \exp \left(-k_{l m} S^{(j)}(x, y)\right)\right] \cos \left(k_{l} x\right) \cos \left(k_{m} y\right)
\end{aligned}
$$

$$
\begin{aligned}
\sigma^{(j)} \sum_{l, m} & \left\{k _ { l m } \left[A_{l m}^{(j)} \exp \left(k_{l m} S^{(j)}(x, y)\right)\right.\right. \\
& \left.-B_{l m}^{(j)} \exp \left(-k_{l m} S^{(j)}(x, y)\right)\right] \cos \left(k_{l} x\right) \cos \left(k_{m} y\right) \\
& +k_{l} \frac{\partial S^{(j)}(x, y)}{\partial x}\left[A_{l m}^{(j)} \exp \left(k_{l m} S^{(j)}(x, y)\right)\right. \\
& \left.+B_{l m}^{(j)} \exp \left(-k_{l m} S^{(j)}(x, y)\right)\right] \sin \left(k_{l} x\right) \cos \left(k_{m} y\right) \\
& +k_{m} \frac{\partial S^{(j)}(x, y)}{\partial y}\left[A_{l m}^{(j)} \exp \left(k_{l m} S^{(j)}(x, y)\right)\right. \\
& \left.\left.+B_{l m}^{(j)} \exp \left(-k_{l m} S^{(j)}(x, y)\right)\right] \cos \left(k_{l} x\right) \sin \left(k_{m} y\right)\right\} \\
= & \sigma^{(j+1)} \sum_{l, m}\left\{k _ { l m } \left[A_{l m}^{(j+1)} \exp \left(k_{l m} S^{(j)}(x, y)\right)\right.\right. \\
& \left.-B_{l m}^{(j+1)} \exp \left(-k_{l m} S^{(j)}(x, y)\right)\right] \cos \left(k_{l} x\right) \cos \left(k_{m} y\right) \\
& +k_{l} \frac{\partial S^{(j)}(x, y)}{\partial x}\left[A_{l m}^{(j+1)} \exp \left(k_{l m} S^{(j)}(x, y)\right)\right. \\
& \left.+B_{l m}^{(j+1)} \exp \left(-k_{l m} S^{(j)}(x, y)\right)\right] \sin \left(k_{l} x\right) \cos \left(k_{m} y\right) \\
& +k_{m} \frac{\partial S^{(j)}(x, y)}{\partial y}\left[A_{l m}^{(j+1)} \exp \left(k_{l m} S^{(j)}(x, y)\right)\right. \\
& \left.\left.+B_{l m}^{(j+1)} \exp \left(-k_{l m} S^{(j)}(x, y)\right)\right] \cos \left(k_{l} x\right) \sin \left(k_{m} y\right)\right\} \\
&
\end{aligned}
$$

Projecting these equations onto sine and cosine basis finally lead to expressions (6)-(9). On the other hand, as the potential should not diverge when $z \rightarrow \infty$, Eq. (11) is obtained.

\section{References}

Jupp, D. L. B. and K. Vozoff, Stable iterative methods for the inversion of geophysical data, Geophys. J. Roy. Astr. Soc., 42, 957, 1975.

Loke, M. H. and R. D. Barker, Rapid least-squares inversion of apparent resistivity pseudosections by a quasi-Newton method, Geophys. Prosp., 44, 131-152, 1996a.

Loke, M. H. and R. D. Barker, Practical techniques for 3D resistivity surveys and data inversion, Geophys. Prosp., 44, 499-523, $1996 \mathrm{~b}$.

Monteiro Santos, F., A. Dupis, A. Andrade Afonso, and L. Mendes Victor, $1 \mathrm{D}$ joint inversion of AMT and resistivity data acquired over a graben, $J$. Appl. Geophys., 38, 115-129, 1997.

Mundry, E., Geoelectrical model calculations for two-dimensional resistivity distributions, Geophys. Prosp., 32, 124-131, 1984.

Noel, M. and B. Xu, Archaeological investigation by electrical resistivity tomography: a preliminary study, Geophys. J. Int., 107, 95-102, 1991.

Oldenburg, D. W. and Y. Li, Inversion of induced polarization data, Geophysics, 59, 1327-1341, 1994.

Oldenburg, D. W., P. R. McGillivary, and R. G. Ellis, Generalized subspace method for large scale inverse problems, Geophys. J. Int., 114, 12-20, 1993. 
Osella, A. and P. Martinelli, Magnetotelluric response of anisotropic 2D structures, Geophys. J. Int., 115, 819-828, 1993.

Osella, A., A. Favetto, P. Martinelli, and D. Cernadas, Electrical imaging of an alluvial aquifer at the Antinaco-Los Colorados valley in the Sierras Pampeanas, Argentina, J. Appl. Geophys., 41(4), 359-368, 1999.

Osella, A., P. Martinelli, and D. Cernadas, 2D geoelectrical modeling using a Rayleigh-Fourier method, IEEE Trans. on Geoscience and Remote Sensing, 38(3), 1-8, 2000.

Park, S. K. and G. P. Van, Inversion of pole-pole data for 3-D resistivity structure beneath arrays of electrodes, Geophysics, 56, 951-960, 1991.

Pous, J., P. Queralt, and R. Chávez, Lateral and topographic effects in geoelectrical soundings, J. Appl. Geophys., 35, 237-248, 1996.

Queralt, P., J. Pous, and A. Marcuello, 2D resistivity modeling: An approach to array parallel to the strike direction, Geophysics, 56, 941-950,
1991.

Schulz, R. and B. Tezkan, Interpretation of resistivity measurements over two-dimensional structures, Geophys. Prosp., 36, 962-975, 1988.

Spitzer, K., 3D finite difference algorithm for DC resistivity modeling using conjugate gradient methods, Geophys. J. Int., 123, 903-914, 1995.

Van Nostrand, R. G. and K. L. Cook, Discussion of 'Apparent resistivity for dipping beds', by Maeda, K. (GEO-20-01-0123-0147), Geophysics, 20, 140-143, 1955.

Zhang, J., R. L. Mackie, and T. R. Madden, 3-D resistivity forward modeling and inversion using conjugate gradients, Geophysics, 60, 1313-1325, 1995

N. Bonomo, A. Osella (e-mail: osella@df.uba.ar), and P. Martinelli 